\title{
Tunneling of Massive Vector Particles from Types of BTZ-like Black Holes
}

\author{
Wajiha Javed, ${ }^{1, *}$ Riasat Ali, $^{2,+}$ Rimsha Babar, $^{1, \ddagger}$ and Ali Övgün ${ }^{3,4}, \S$ \\ ${ }^{1}$ Division of Science and Technology, University of Education, Township-Lahore, Pakistan \\ ${ }^{2}$ Department of Mathematics, Government College, University Faisalabad Layyah Campus, Layyah-31200, Pakistan \\ ${ }^{3}$ Instituto de Física, Pontificia Universidad Católica de Valparaíso, Casilla 4950, Valparaíso, Chile \\ ${ }^{4}$ Physics Department, Faculty of Arts and Sciences, Eastern Mediterranean University, Famagusta, North Cyprus, via Mersin 10, Turkey \\ (Dated: April 3, 2019)
}

\begin{abstract}
In this paper, we analyze the Hawking radiation phenomenon for types of Banados-TeitelboimZanelli-like (BTZ-like) black holes. For this purpose, using the Hamilton-Jacobi method, we consider semi-classical WKB approximation to calculate the tunneling probabilities of massive boson particles. For these particles, we use the equation of motion for the Glashow-Weinberg-Salam model. Using quantum tunneling process of charged massive bossons, we compute the corresponding Hawking temperatures. Furthermore, we discuss the effects of rotation parameter on tunneling probability and temperature.
\end{abstract}

Keywords: Proca equation; Bosons particles; Hawking radiation; Black hole thermodynamics; Higherdimensional black holes.

\section{INTRODUCTION}

The hypothesis of general relativity (GR) suggested that a sufficiently compact mass can change the shape of spacetime fabric for configuration of a black hole (BH). Hawking established that quantum events reserved $\mathrm{BH}$ to emit radiation. These radiation emit from a $\mathrm{BH}$ are just like a black body radiation and known as Hawking radiation [1]. The Hawking radiation process is studied by creation of a couple of positive and negative charged energy particles in the vicinity of the horizon of BH. The positive-energy particle escape out from horizon, causes Hawking radiation while the negative-energy particle of this pair falls inside the $\mathrm{BH}$. The rate at which particles overcome the horizon can be calculated by quantum tunneling phenomenon. According to this technique particles have finite tunneling probability denoted by $\tilde{\Gamma}$ to cross the event horizon, which depends upon the imaginary part of particle's action $[2,3]$. The probability $\tilde{\Gamma}$ for emitted particles can be defined as

$$
\tilde{\Gamma} \propto \exp \left[-\frac{2}{\hbar} \operatorname{Im}\right],
$$

where $£$ denotes the semi-classical particle's action and $\hbar$ denotes the Planck's constant.

The study of Hawking temperature by utilizing quantum tunneling process from various BHs has attracted lots of researchers. Different efforts have been made to calculate this radiation spectrum by considering quantum mechanics of fermions, bosons, scalars, Dirac and photon particles etc. Various authors [4-30] analyzed vector particles, fermionic particles, spin-2 and spin-3/2 particles tunneling to study the Hawking radiation phenomenon for different BHs and wormholes. The tunneling probabilities for Kerr-Newman BH [31] and charged black string [32] have also been investigated. In order to study Hawking temperature by using Kerner and Mann's formulation, Sharif and Javed [33] studied the fermions tunneling phenomenon through the horizons of charged anti-de Sitter BHs, charged torus-like BHs, Plebański - Demianski family of BHs, regular BHs and and traversable wormholes. The same authors [34] discussed the Hawking radiation phenomenon as fermions tunneling for a pair of charged accelerating and rotating BHs with NUT parameter. They have calculated the corresponding Hawking temperature. Sharif and Javed [35] have also investigated the quantum corrections for regular BHs, i.e., Bardeen and ABGB. Javed, Abbas and Ali discussed charged vector particles tunneling process for a pair of accelerating and rotating BHs as well as for 5D gauged super-gravity BHs [36]. The Hawking radiation for charged NUT (Newman-Unti-Tamburino) BH having rotation and acceleration parameters has been viewed in Ref.[37].

\footnotetext{
*Electronic address: wajiha.javed@ue.edu.pk; wajihajaved84@yahoo.com

${ }^{\dagger}$ Electronic address: riasatyasin@gmail.com

‡Electronic address: rimsha.babar10@gmail.com

§Electronic address: ali.ovgun@pucv.cl; https: / / www . aovgun.com
} 
The tunneling rate for outgoing particles is found by utilizing the imaginary component of particle's action. Övgün, Javed and Ali [38] found the tunneling rate of charged massive bosons for various types of BHs surrounded by the perfect fluid in Rastall theory. Several efforts [39]-[43] have been made to analyze the tunneling process of charged and uncharged scalar and Dirac particles for different BHs. The tunneling rate of spin- $\frac{1}{2}$ particles through horizon of Rindler spacetime was discussed as well as the Unruh temperature was analyzed [44]. Kraus and Wilczek $[45,46]$ visualized the semi-classical method to study the Hawking radiation as a tunneling through the horizon. This method contains the calculation for the process of s-wave emission across the horizon.

In continuation of previous work, in this paper we calculate the electromagnetic boson particles radiation, emitted by BTZ-like BHs. For this analysis, we study the tunneling of massive vector bosons through event horizons of higher dimensional charged BTZ-like BHs by using semi-classical approximation, also we calculate the corresponding Hawking temperatures. Charged vector particles (spin-1) such as charged $W^{ \pm}$-bosons having much importance in Standard Model. In the background of BHs geometries; the behavior of the bosons particles can be traced out by using Proca equation. Here, we analyze the field equations of charged $W^{ \pm}$-bosons by applying the Lagrangian of Glashow-Weinberg-Salam model [47].

This paper is organized as follows: In Section II, we analyze the tunneling probability $\tilde{\Gamma}$ of boson particles and the corresponding Hawking temperature $T_{H}$ for charged BTZ magnetic static BH, by calculating the radial part of particle's action for massive bosons $W^{ \pm}$. Section III presents the vector particles tunneling for rotating BTZ magnetic BH. Section IV provides the results of $\tilde{\Gamma}$ and $T_{H}$ for $(n+1)$-dimensional BTZ magnetic BHs with rotation parameter. Section $\mathbf{V}$ contains the results of tunneling probability and Hawking temperature for $(n+1)$-dimensional BTZ magnetic BHs with more than one rotation parameters. Section VI summarizes all the results of the paper.

\section{CHARGED BTZ MAGNETIC STATIC BLACK HOLE}

Black holes play a very essential part in the field of General Relativity. After the discovery of first Schwarzschild $\mathrm{BH}$ many extensions have been made by introducing the rotation, charge, acceleration, cosmological, quintessential and many other parameters. All these extensions of BHs are associated with D-branes. The BTZ solution in $(2+1)$-dimensional space presents a valuable template to guide people in order to analyze concepts of quantum gravitational problems and AdS/CFT conjecture. In a charged $\mathrm{BH}$ the electric field is related to the temporal factor $A_{t}$ of gauged potential whereas the magnetic field is related to the angular component $A_{\phi}$. As a result, one can expect the magnetic solution to be written in a metric gauge, in which the $g_{t t}$ and $g_{\phi \phi}$ components are relatively exchanged for their effects to those used in electric gauge to describe $\mathrm{BH}$ solutions [48]. The motivation of our work is that we are looking for a charged $\mathrm{BH}$ with magnetic field instead of $\mathrm{BH}$ interpretation with the electrical field.

The background geometry of BTZ BH can be studied for more generalized, i.e., $n$ and $(n+1)$-dimensional spaces and it can be used to explain the effects of more rotation parameters involve in $\mathrm{BH}$ to investigate either they will effect or not the tunneling spectrum of Hawking radiation. There have been a lot of work on quantum tunneling spectrum for charged BTZ BH but this form of BTZ-like BH is more generalized than the BTZ BH. The line-element of this $\mathrm{BH}$ is given by [49]

$$
d s^{2}=-\frac{r^{2}}{l^{2}} d t^{2}+\frac{1}{R(r)} d r^{2}+l^{2} R(r) \gamma^{2} d \phi^{2},
$$

where $r$ is radial coordinate, $l$ denotes the curvature radius associated with negative cosmological constant $\Lambda=-\frac{1}{l^{2}}$, $R(r)$ represents the metric function which is given by

$$
R(r)=\frac{r^{2}}{l^{2}}-\left[M+8 q^{2} l^{2} \gamma^{2} \ln \left(\frac{r}{l}\right)\right],
$$

where $M$ and $q$ indicate the mass and charge of BTZ solution, while $\gamma$ is arbitrary parameter.

It is to be noted that the above metric (2) after applying local transformation $t \rightarrow i l \gamma \phi$ and $\phi \rightarrow i t / l$ will convert to the static 3D Schwarzschild metric. So, we can change the role of $t$ and $\phi$ co-ordinates by using given transformation. The Eq.(2) can be rewritten as

$$
d s^{2}=X(r) d t^{2}+Y(r) d r^{2}+Z(r) d \phi^{2},
$$

where the radial functions $X(r), Y(r)$ and $Z(r)$ are expressed as

$$
X(r)=-\frac{r^{2}}{l^{2}}, Y(r)=\frac{1}{R(r)}, Z(r)=l^{2} R(r) \gamma^{2}
$$


The gauge potential of this $\mathrm{BH}$ can be defined as

$$
A_{\mu}=-2 q l^{2} \gamma^{2} h(r) \delta_{\mu}^{\phi}
$$

where the only non-zero component is $A_{\phi}$ due to magnetic field and $h(r)$ is an arbitrary function of $r$.

In order to calculate the tunneling rate for massive vector particles through the $\mathrm{BH}^{\prime}$ s event horizon $r_{+}$, we will consider the relativistic wave equation, i.e., Proca equation with electromagnetic effects. The motion of charged massive spin-1 fields is traced out by the given Proca equation in the background of electromagnetic field $\Psi_{\mu}$ [36]

$$
\frac{1}{\sqrt{-\mathbf{g}}} \partial_{\mu}\left(\sqrt{-\mathbf{g}} \Psi^{v \mu}\right)+\frac{m^{2}}{h^{2}} \Psi^{\nu}+\frac{\iota}{h} e A_{\mu} \Psi^{v \mu}+\frac{\iota}{h} e F^{v \mu} \Psi_{\mu}=0,
$$

here $g$ indicates the determinant of the coefficient matrix, $e$ is particle charge and $\Psi^{\mu \nu}$ is anti-symmetric tensor which can be defined as

$$
\Psi_{v \mu}=\partial_{\nu} \Psi_{\mu}-\partial_{\mu} \Psi_{v}+\frac{\iota}{h} e A_{v} \Psi_{\mu}-\frac{\iota}{h} e A_{\mu} \Psi_{v} \text { and } F^{\mu v}=d^{\mu} A^{v}-d^{v} A^{\mu}
$$

where, $A_{\mu}$ is the electromagnetic vector potential related to $\mathrm{BH}$ and $d^{\mu}$ refers to the geometrical covariant derivative, $m$ represents the particle's mass. The non-zero components of $\Psi^{\mu}$ and $\Psi^{v \mu}$ are given as follows

$$
\begin{aligned}
\Psi^{0} & =\frac{\Psi_{0}}{X(r)}, \quad \Psi^{1}=\frac{\Psi_{1}}{Y(r)}, \quad \Psi^{2}=\frac{\Psi_{2}}{Z(r)}, \\
\Psi^{01} & =\frac{\Psi_{01}}{X(r) Y(r)}, \quad \Psi^{02}=\frac{\Psi_{02}}{X(r) Z(r)}, \quad \Psi^{12}=\frac{\Psi_{12}}{Y(r) Z(r)} .
\end{aligned}
$$

Using Hamilton-Jacobi ansatz, the wave function in terms of semi-classical particle's action $£$ by applying the WKB approximation can be defined as [36, 37], [50]-[52]

$$
\Psi_{v}=c_{v} \exp \left[\frac{\iota}{\hbar} £_{0}(t, r, \phi)+\sum_{i=1}^{n} \hbar^{i} £_{i}(t, r, \phi)\right]
$$

Using Eq.(8) in the wave Eq.(7) for $i=1,2,3, \ldots$ and by neglecting the higher order terms, we get the following set of equations

$$
\begin{aligned}
& \frac{1}{Y(r)}\left[c_{1}\left(\partial_{0} £_{0}\right)\left(\partial_{1} £_{0}\right)-c_{0}\left(\partial_{1} £_{0}\right)^{2}\right]+\frac{1}{Z(r)}\left[c_{2}\left(\partial_{0} £_{0}\right)\left(\partial_{2} £_{0}\right)-c_{0}\left(\partial_{2} £_{0}\right)^{2}\right. \\
& \left.-e A_{\phi} c_{0}\left(\partial_{2} £_{0}\right)\right]+\frac{e A_{\phi}}{Z(r)}\left[c_{2}\left(\partial_{0} £_{0}\right)-e A_{\phi} c_{0}-c_{0}\left(\partial_{2} £_{0}\right)\right]-m^{2} c_{0}=0 \\
& \frac{1}{X(r)}\left[c_{0}\left(\partial_{0} £_{0}\right)\left(\partial_{1} £_{0}\right)-c_{1}\left(\partial_{0} £_{0}\right)^{2}\right]+\frac{1}{Z(r)}\left[c_{2}\left(\partial_{1} £_{0}\right)\left(\partial_{2} £_{0}\right)-c_{1}\left(\partial_{2} £_{0}\right)^{2}\right. \\
& \left.-e A_{\phi} c_{1}\left(\partial_{2} £_{0}\right)\right]+\frac{e A_{\phi}}{Z(r)}\left[c_{2}\left(\left(\partial_{1} £_{0}\right)-e A_{\phi}\right) c_{1}-c_{1}\left(\partial_{2} £_{0}\right)\right]-m^{2} c_{1}=0 \\
& \frac{1}{X(r)}\left[c_{0}\left(\partial_{0} £_{0}\right)\left(\partial_{2} £_{0}\right)-c_{2}\left(\partial_{0} £_{0}\right)^{2}+e A_{\phi}\left(\partial_{0} £_{0}\right) c_{0}\right]+\frac{1}{Y(r)}\left[c_{1}\left(\partial_{1} £_{0}\right)\right. \\
& \left.\left(\partial_{2} £_{0}\right)-c_{2}\left(\partial_{1} £_{0}\right)^{2}+e A_{\phi} c_{1}\left(\partial_{1} £_{0}\right)\right]-m^{2} c_{2}=0 .
\end{aligned}
$$

Using separation of variables technique, one can write the particle's action in the following form

$$
£_{0}=-(E-j \omega) t+G(r)+k \phi
$$

where $E, \omega$ and $j$ represent particle's energy, angular velocity and angular momentum, respectively. Using Eqs.(9)(11), the matrix equation can be written as

$$
\Xi\left(c_{0}, c_{1}, c_{2}, c_{3}\right)^{T}=0
$$


where $\Xi$ is a $3 \times 3$ ordered matrix and its components are given below

$$
\begin{aligned}
& \Xi_{00}=-\frac{\dot{G}^{2}}{Y(r)}-\frac{1}{Z(r)}\left(k^{2}-e A_{\phi} k\right)-m^{2}-\frac{e A_{\phi}}{Z(r)}\left(k+e A_{\phi}\right), \\
& \Xi_{01}=-\frac{1}{Y(r)} \dot{G}(E-j \omega), \\
& \Xi_{02}=-\frac{1}{Z(r)}(E-j \omega) k-\frac{e A_{\phi}}{Z(r)}(E-j \omega), \\
& \Xi_{10}=-\frac{1}{X(r)}(E-j \omega) \dot{G}, \\
& \Xi_{11}=-\frac{1}{X(r)}(E-j \omega)^{2}-\frac{1}{Z(r)}\left(k^{2}+e A_{\phi} k\right)-m^{2}-\frac{e A_{\phi}}{Z(r)}\left(k+e A_{\phi}\right), \\
& \Xi_{12}=\frac{1}{Z(r)} \dot{G} k+\frac{e A_{\phi}}{Z(r)} \dot{G}, \\
& \Xi_{20}=-\frac{1}{X(r)}\left[(E-j \omega) k+e A_{\phi}(E-j \omega)\right], \\
& \Xi_{21}=\frac{1}{Y(r)}\left[\dot{G} k+e A_{\phi} \dot{G}\right], \\
& \Xi_{22}=-\frac{1}{X(r)}\left[(E-j \omega)^{2}+e A_{\phi}(E-j \omega)\right]-\frac{1}{Y(r)} \dot{G}^{2}-m^{2},
\end{aligned}
$$

where $\dot{G}=\partial_{r} £_{0}$ and $k=\partial_{\phi} £_{0}$. For the non-trivial solution, we take $|\Xi|=0$ and get

$$
\dot{G}^{2}(r)=\frac{\left(E-e A_{\phi}-j \omega\right)^{2}+X}{\frac{-Z(r)}{Y(r)}},
$$

where

$$
X=(E-j \omega) k+k^{2}+m^{2}-e A_{\phi} k
$$

Integrating around the pole at the horizon, Eq.(13) provides

$$
\operatorname{Im} G^{ \pm}(r)= \pm \iota \pi \frac{\left(E-j \omega-e A_{\phi}\right)}{2 \tilde{\kappa}_{\left(r_{+}\right)}},
$$

where $G^{+}$and $G^{-}$represent radial function for the outgoing and incoming particles, respectively following the radial path. Therefore, particle's action $£$ is reduce to its radial part $G$. The surface gravity $\tilde{\kappa}\left(r_{+}\right)$of this BH is given as follows

$$
\tilde{\kappa}\left(r_{+}\right)=\frac{\sqrt{r_{+}^{2}-4 q^{2} \gamma^{2} l^{4}}}{l^{2}} .
$$

Using Eq.(1), the tunneling probability $\tilde{\Gamma}$ for boson particles can be defined as ratio of probabilities of emission and absorption, i.e.,

$$
\begin{aligned}
\tilde{\Gamma} & =\frac{\tilde{\Gamma}_{(\text {emission })}}{\tilde{\Gamma}_{(\text {absorption })}}=\frac{\exp \left[-\frac{2}{\hbar}\left(\operatorname{Im} G^{+}\right)\right]}{\exp \left[-\frac{2}{\hbar}\left(\operatorname{Im} G^{-}\right)\right]}=\exp \left[-\frac{4}{\hbar} \operatorname{Im} G^{+}\right] \\
& =\exp \left[-2 \pi \frac{l^{2}\left(E-j \omega-e A_{\phi}\right)}{\sqrt{r_{+}^{2}-4 q^{2} \gamma^{2} l^{4}}}\right] \quad(\text { for } \hbar=1) .
\end{aligned}
$$

Using Boltzmann factor $\tilde{\Gamma}_{B}=\exp \left[\left(E-j \omega-e A_{\phi}\right) / T_{H}\right]$, the corresponding Hawking temperature can be calculated as

$$
\tilde{T}_{H}=\frac{\sqrt{r_{+}^{2}-4 q^{2} \gamma^{2} l^{4}}}{4 \pi l^{2}}
$$


The tunneling probability of boson particles depending upon charged potential of BH, particle's charge, energy and angular momentum, the curvature radius $\ell$ and $\mathrm{BH}$ radius. While, the Hawking temperature is depending upon charge of $\mathrm{BH}$, the curvature radius and $\mathrm{BH}$ radius.

\section{ROTATING BTZ MAGNETIC BLACK HOLE}

In this section, we analyze the metric by using co-ordinate transformation such as $t \longrightarrow \lambda t-a \varphi$ and $\varphi \longrightarrow \lambda \varphi-\frac{a}{l} t$. One can obtain the BTZ BH with rotation parameter as well as magnetic charge. The line-element of this BH is given by [49]

$$
d s^{2}=-\frac{r^{2}}{l^{2}}(\lambda d t-a d \varphi)^{2}+\frac{1}{R(r)} d r^{2}+l^{2} R(r) \gamma^{2}\left(\frac{a}{l^{2}} d t-\lambda d \varphi\right)^{2}
$$

where $\lambda=\sqrt{1+\frac{a^{2}}{l^{2}}}$ and $a$ is a rotation parameter. The gauge potential $A_{\mu}$ for this $\mathrm{BH}$ is given by

$$
A_{\mu}=-2 q l \gamma^{2} h(r)\left(\frac{a}{l} \delta_{\mu}^{t}-\lambda l \delta_{\mu}^{\varphi}\right)
$$

The non-zero components are $A_{t}$ and $A_{\varphi}$.

Following the procedure given in the preceding Section II, we can obtain the surface gravity $\tilde{\kappa}\left(r_{+}\right)$of rotating BTZ-like magnetic $\mathrm{BH}$ in the following form

$$
\tilde{\kappa}\left(r_{+}\right)=\sqrt{\frac{\lambda^{2} r_{+}^{2}}{l^{3}}-\frac{r_{+}^{2} \gamma^{2} a^{2}}{l^{2}}+4 q^{2} \gamma^{4} r_{+} a^{2}-\frac{4 \lambda^{2} q^{2} \gamma^{2}}{l^{2}}+4 q^{2} l^{2} \gamma^{4} a^{2}-\frac{16 l^{2} q^{4} \gamma^{6} a^{2}}{r_{+}}} .
$$

The tunneling probability $\tilde{\Gamma}$ for this case of BTZ BH can be obtained as

$$
\tilde{\Gamma}=\exp \left[\frac{-2 \pi\left(E-j \omega-e A_{t}-e A_{\varphi}\right)}{\sqrt{\frac{\lambda^{2} r_{+}^{2}}{l^{3}}-\frac{r_{+}^{2} \gamma^{2} a^{2}}{l^{2}}+4 q^{2} \gamma^{4} r_{+} a^{2}-\frac{4 \lambda^{2} q^{2} \gamma^{2}}{l}+4 q^{2} l \gamma^{4} a^{2}-\frac{16 l^{2} q^{4} \gamma^{6} a^{2}}{r_{+}}}}\right] .
$$

Using Boltzmann factor, we can obtained the corresponding Hawking temperature $\tilde{T}_{H}$ for boson particles as

$$
\tilde{T}_{H}=\sqrt{\frac{1}{\pi^{2}}\left[\frac{\lambda^{2} r_{+}^{2}}{4 l^{3}}-\frac{r_{+}^{2} \gamma^{2} a^{2}}{4 l^{2}}+q^{2} \gamma^{4} r_{+} a^{2}-\frac{\lambda^{2} q^{2} \gamma^{2}}{l^{2}}+q^{2} l^{2} \gamma^{4} a^{2}-\frac{4 l^{2} q^{4} \gamma^{6} a^{2}}{r_{+}}\right]}
$$

Thus, the surface gravity $\tilde{\kappa}\left(r_{+}\right)$, tunneling rate $\tilde{\Gamma}$ and Hawking temperature $\tilde{T}_{H}$, all are depending upon rotation parameter.

\section{IV. $(n+1)$-DIMENSIONAL CHARGED BTZ BLACK HOLE}

In this section, we calculate the massive boson particles tunneling probability at the outer horizon of the $(n+1)$ dimensional BTZ magnetic BH with rotation parameter. The line-element of this BH is given by [49]

$$
\begin{aligned}
d s^{2} & =-\frac{r^{2}}{l^{2}}(\lambda d t-a d \varphi)^{2}+\frac{1}{R(r)} d r^{2}+l^{2} R(r) \gamma^{2}\left(\frac{a}{l^{2}} d t-\lambda d \varphi\right)^{2}+r^{2} d \phi^{2} \\
& +\frac{r^{2}}{l^{2}} \sum_{i=1}^{n-3} d x^{i} .
\end{aligned}
$$

It is to be noted that the $x^{i}$ is the dimension length of $\mathrm{BH}$ and the angular parameter $\varphi$ and $\phi$ are dimensionless for $[0,2 \pi]$. For this case, the metric function $R(r)$ is defined as

$$
R(r)=\frac{r^{2}}{l^{2}}-\frac{1}{r^{d-3}}\left[M+2^{(d-1) / 2}(2 q l \gamma)^{d-1} \ln \left(\frac{r}{l}\right)\right]
$$


Using the same formalism as defined earlier in preceding Section II for this line-element, the surface gravity $\tilde{\kappa}\left(r_{+}\right)$ can be obtained as

$$
\tilde{\kappa}\left(r_{+}\right)=\sqrt{\frac{\lambda^{2} \hat{R}\left(r_{+}\right) r_{+}}{2 l^{2}}+\frac{\dot{R}^{2}\left(r_{+}\right) a^{2} \gamma^{2}}{4}},
$$

where $\hat{R}(r)$ is defined as

$$
\begin{aligned}
\hat{R}\left(r_{+}\right) & =\frac{2 r_{+}}{l^{2}}-\frac{M(3-d)}{r_{+}^{d-2}}+\frac{(3-d)}{r_{+}^{d-2}} 2^{(d-1) / 2}(2 q l \gamma)^{d-1} \ln \left(\frac{r_{+}}{l}\right) \\
& +\frac{2^{(d-1) / 2}(2 q l \gamma)^{d-1}}{r_{+}^{d-2}} .
\end{aligned}
$$

The tunneling rate $\tilde{\Gamma}$ of boson particles at horizon can be calculated as follows

$$
\tilde{\Gamma}=\exp \left[\frac{-2 \pi l\left(E-j \omega-e A_{\mu}\right)}{\sqrt{2 \lambda^{2} \hat{R}\left(r_{+}\right) r_{+}+l^{2} \hat{R}^{2}\left(r_{+}\right) a^{2} \gamma^{2}}}\right] \text {. }
$$

The corresponding Hawking temperature $\tilde{T}_{H}$ is given by

$$
\tilde{T}_{H}=\frac{\sqrt{2 \lambda^{2} \hat{R}\left(r_{+}\right) r_{+}+\dot{R}^{2}\left(r_{+}\right) l^{2} a^{2} \gamma^{2}}}{4 \pi l} .
$$

The tunneling rate $\tilde{\Gamma}$ and Hawking temperature $\tilde{T}_{H}$ of a boson particles depend on rotation parameter, mass and $\mathrm{BH}$ radius.

\section{V. $(n+1)$-DIMENSIONAL CHARGED BTZ BLACK HOLE WITH $[n / 2]$ ROTATION PARAMETERS}

This section is devoted to analyze the massive boson particles tunneling probability at the outer horizon of the metric of $(n+1)$-dimension $\mathrm{BH}$ with more rotation parameters and charge. The line-element of this $\mathrm{BH}$ is given by [49]

$$
\begin{aligned}
d s^{2}= & -\frac{r^{2}}{l^{2}}\left[\lambda d t-\sum_{i=1}^{k} a_{i} d \varphi^{i}\right]^{2}+\frac{1}{R(r)} d r^{2}+R(r) \gamma^{2}\left[\sqrt{\lambda^{2}-1} d t-\frac{\lambda}{\sqrt{\lambda^{2}-1}}\right. \\
& \left.\sum_{i=1}^{k} a_{i} d \varphi^{i}\right]^{2}+\frac{r^{2}}{l^{2}\left(\lambda^{2}-1\right)} \sum_{i<j}^{k}\left[a_{i} d \varphi_{j}-a_{j} d \varphi_{i}\right]^{2}+\frac{r^{2}}{l^{2}} d X^{2} .
\end{aligned}
$$

There are $[n / 2]$ independent rotation parameters. It is to be noted that $\lambda=\sqrt{1+\sum_{i=1}^{k} \frac{a_{i}^{2}}{l^{2}}}$ and $d X^{2}$ is the Euclidean metric on the $(n-k-2)$-dimensional subspace. The function $R(r)$ is defined as

$$
R(r)=\frac{r^{2}}{l^{2}}-\frac{1}{r^{d-3}}\left[M+2^{(d-1) / 2}(2 q l \gamma)^{d-1} \ln \left(\frac{r}{l}\right)\right] .
$$

Following the same procedure given in the preceding sections, the surface gravity $\tilde{\kappa}\left(r_{+}\right)$of $(n+1)$-dimensional BTZ-like $\mathrm{BH}$ with more rotation parameter can be calculated as

$$
\tilde{\kappa}\left(r_{+}\right)=\sqrt{\frac{\lambda^{2} \hat{R}\left(r_{+}\right) r_{+}}{2 l^{2}}+\frac{\dot{R}^{2}\left(r_{+}\right)\left(\lambda^{2}-1\right) \gamma^{2}}{4}} .
$$

The tunneling rate $\tilde{\Gamma}$ of boson particles at event horizon can be calculated as

$$
\tilde{\Gamma}=\exp \left[\frac{-2 \pi l\left(E-j \omega-e A_{\mu}\right)}{\sqrt{2 \lambda^{2} \hat{R}\left(r_{+}\right) r_{+}+l^{2} \hat{R}^{2}\left(r_{+}\right)\left(\lambda^{2}-1\right) \gamma^{2}}}\right] .
$$


The corresponding Hawking temperature $\tilde{T}_{H}$ can be deduced as

$$
\tilde{T}_{H}=\frac{\sqrt{2 \lambda^{2} \hat{R}\left(r_{+}\right) r_{+}+\hat{R}^{2}\left(r_{+}\right) l^{2}\left(\lambda^{2}-1\right) \gamma^{2}}}{4 \pi l}
$$

where

$$
\begin{aligned}
\hat{R}\left(r_{+}\right) & =\frac{2 r_{+}}{l^{2}}-\frac{M(3-d)}{r_{+}^{d-2}}+\frac{(3-d)}{r_{+}^{d-2}} 2^{(d-1) / 2}(2 q l \gamma)^{d-1} \ln \left(\frac{r_{+}}{l}\right) \\
& +\frac{2^{(d-1) / 2}(2 q l \gamma)^{d-1}}{r_{+}^{d-2}} .
\end{aligned}
$$

It is to be noted that $\lambda=\sqrt{1+\sum_{i=1}^{k} \frac{a_{i}^{2}}{l^{2}}}$ depends upon more rotation parameters. Hence, the surface gravity, tunneling probability and the Hawking temperature for vector particles are depending upon more rotation parameters. The Hawking temperature increases due to rotation parameter.

\section{CONCLUSIONS}

In this work, we have studied the massive boson particles tunneling phenomenon from 3-dimensional static charged BTZ magnetic as well as rotating BH and $(n+1)$-dimensional charged BTZ BH with rotation parameter. We extended our analysis to higher-dimensional rotating charged BTZ-like BHs. For this purpose, initially we have utilized the equation of motion for massive bosons by generalizing the Proca wave equation of charged particles in curved spacetime in the background of electromagnetic field. We have applied the WKB approximation to the Proca equation and in result we have obtained a set of field equations, then we have used the separation of variables technique to solve these equations. We have obtained the radial function of semi-classical particle's action by putting the determinant of coefficient matrix equals to zero. By utilizing the obtained surface gravity, we have calculated the tunneling probability and Hawking temperature for all types of given BTZ-like BHs.

The tunneling probabilities and Hawking temperatures are depending upon the parameters on which BHs are dependent. It is interesting to specify here that the back-reaction impacts of the radiated particles on the BH geometry and self-gravitating effects have been ignored and the determined Hawking temperature is just a main term and turned out to be reliable with $\mathrm{BH}$ universality.

Our analysis expressed that if the effect of rotation parameter is viewed, the action of the tunneling boson particles on the event horizon will be different from the real particle's action and the calculated tunneling probabilities are not just depending on the boson particles's energy $E$, angular momentum $j$ and particle's charge $e$ but it is also depending upon the gauge potential $A_{\mu}$ of $\mathrm{BH}$, rotation parameter $a$, curvature radius $\ell$ and $\mathrm{BH}$ event horizon $r_{+}$. Moreover, the corresponding Hawking temperature depending upon the charge $q$, rotation parameter, curvature radius and event horizon of BHs. Moreover, the Hawking temperature of $\mathrm{BH}$ increases due to the rotation parameter. Furthermore, our analysis is similar to the analysis for charged scalar and fermion particles tunneling from these BHs. The charged scalar and fermion particles radiate through the BHs horizons with the same energy.

\section{Acknowledgments}

A. Ö. acknowledges financial support provided under the Chilean FONDECYT Grant No. 3170035.

[1] R. Mario, Physics. Essays. 12, 346(2000).

[2] S. W. Hawking, Nature 248, 30(1974).

[3] S. W. Hawking, Commun. Math. Phys. 43, 199(1975).

[4] I. Sakalli and A. Övgün, Gen. Rel. Grav. 48, no. 1, 1 (2016).

[5] I. Sakalli and A. Ovgun, Eur. Phys. J. Plus 130, no. 6, 110 (2015).

[6] I. Sakalli and A. Ovgun, EPL 110, no. 1, 10008 (2015).

[7] I. Sakalli, A. Övgün and K. Jusufi, Astrophys. Space Sci. 361, no. 10, 330 (2016).

[8] I. Sakalli and A. Ovgun, EPL 118, no. 6, 60006 (2017). 
[9] A. Övgün and K. Jusufi, Eur. Phys. J. Plus 131, no. 5, 177 (2016).

[10] I. Sakalli and A. Ovgun, Astrophys. Space Sci. 359, no. 1, 32 (2015).

[11] I. Sakalli and A. Ovgun, J. Exp. Theor. Phys. 121, no. 3, 404 (2015).

[12] I. Sakalli and A. Övgün, Eur. Phys. J. Plus 131, no. 6, 184 (2016).

[13] A. Övgün, Int. J. Theor. Phys. 55, no. 6, 2919 (2016).

[14] X. M. Kuang, J. Saavedra and A. Övgün, Eur. Phys. J. C 77, no. 9, 613 (2017).

[15] P. A. Gonzalez, A. Övgün, J. Saavedra and Y. Vasquez, Gen. Rel. Grav. 50, no. 6, 62 (2018).

[16] A. Övgün, Adv. High Energy Phys. 2017, 1573904 (2017).

[17] Deyou Chen, Houwen Wu, Haitang Yang, Shuzheng Yang, Int. J. Mod. Phys. A 29 (2014) 1430054.

[18] Deyou Chen, Eur. Phys. J. C 74 (2014) 2687.

[19] G. Gecim and Y. Sucu, Mod. Phys. Lett. A 33, no. 28, 1850164 (2018).

[20] G. Gecim and Y. Sucu, Phys. Lett. B 773, 391 (2017).

[21] G. Gecim and Y. Sucu, Adv. High Energy Phys. 2018, 8728564 (2018).

[22] M.Hossain Ali and K. Sultana, Int.J.Theor.Phys. 56, no.7, 2279-2292 (2017).

[23] M.Hossain Ali, Class.Quant.Grav. 24, 5849-5860 (2007).

[24] M.Hossain Ali, Int.J.Theor.Phys. 47, 2203-2217 (2008).

[25] M.Hossain Ali, Gen.Rel.Grav. 36, 1171-1181 (2004).

[26] H. Pasaoglu and I. Sakalli, Int. J. Theor. Phys. 48, 3517 (2009).

[27] E. T. Akhmedov, V. Akhmedova and D. Singleton, Phys. Lett. B 642, 124 (2006).

[28] X. Q. Li, G.R. Chen, Phys. Lett. B 751, 34(2015).

[29] G. R. Chen, Y.C. Huang, Int. J. Mod. Phys. Rev. A 30, 1550083(2015).

[30] Z. Feng, Y. Chen, X. Zu, Astrophys. space Sci. 48, 359(2015).

[31] T. I. Singh, I. A. Meitei, K. Y. Singh, Astrophys. Space Sci. 361, 103(2015).

[32] K. Jusufi, Ali Övgün, Astrophys. Space Sci. 361, 207(2016)

[33] M. Sharif, W. Javed, Can. J. Phys. 90, 903(2012); ibid. Gen. Relativ. Gravit. 45, 1051(2013); ibid. Can. J. Phys. 91, 43(2013); ibid. J. Exp. Theor. Phys. 115, 782(2012); ibid. Proceedings of the 3rd GalileoXu Guangqi Meeting, Int. J. Mod. Phys.: Conference Series, 23, 271(2013);ibid. Proceedings of the 13th Marcel Grossmann Meeting (Stockholm, 2012), World Scientific, 3, 1950(2015).

[34] M. Sharif, W. Javed, Eur. Phys. J. C72, 1997(2012).

[35] M. Sharif, W. Javed, J. Korean. Phys. Soc. 57, 217(2010).

[36] W. Javed, G. Abbas, R. Ali, Eur. Phys. J. C77, 296(2017).

[37] M. Sharif, W. Javed, Can. J. Phys. 91, 236(2013).

[38] A. Övgün, W. Javed, R. Ali; arXiv:1710.06264.

[39] R. Kerner, R. B. Mann, Phys. Rev. D 73, 104010(2006).

[40] M. H. Dehghani, R. B. Mann, Phys. Rev. D 73, 104003(2006).

[41] D. Ahn, Y. H. Moon, R. B. Mann, I. Fuentes-Schuller JHEP 0806, 062(2008).

[42] R. Kerner, R.B. Mann, Class. Quant. Grav. 25, 095014(2008).

[43] R. Kerner, R.B. Mann, Phys. Lett. B 665, 277(2008).

[44] M. Sharif, W. Javed, J. Exp. Theor. Phys. 115, 782(2012).

[45] P. Kraus, F. Wilczek, Nucl. Phys. B 433, 403(1995).

[46] P. Kraus, F. Wilczek, Nucl. Phys. B 437, 403(1995).

[47] E.D. Commins, P.H. Bucksbaum, Weak Interactions of Leptons and Quarks, Cambridge University Press, Cambridge, 1983.

[48] S. H. Hendi, Class. Quantum Grav. 26, 225014(2009).

[49] S.H. Hendi, Prog. Theor. Phys. 127, 907(2012).

[50] T. Shivalingaswamy, B.A. Kagali, Eur. J. Phys. Education. 2, 1309(2011).

[51] A. Övgün, W. Javed, R. Ali, Advances in High Energy Physics Volume 2018, Article ID 3131620, 11 pages 31620.

[52] X.Q. Li, G.R. Chen, Phys. Lett. B751, 34(2015). 